\title{
Absorption, transport, and metabolism of carotenoids in humans
}

\author{
James Allen 0lson \\ Department of Biochemistry \& Biophysics, Iowa State University, Ames, IA \\ 50011, U.S.A.
}

\begin{abstract}
Vitamin $A$, which is an essential nutrient for humans, may be provided in the diet either as preformed vitamin $A$ or as provitamin $A$ carotenoids. The bioavailability of carotenoids in foods varies greatly, depending on many factors. Carotenoids are emulsified and then are solubilized in micellar form within the intestine with an absolute requirement for conjugated bile salts. Dietary carotenoids are absorbed as part of chylomicra, but in the steady state they are primarily constituents of low density and high density lipoproteins. Carotenoids are metabolized in many different ways, depending on their structure. of particular importance nutritionally is the conversion of provitamin $A$ carotenoids to vitamin A. Carotenoids are found in nearly all tissues, but, particularly, in the liver and adipose tissue. Each carotenoid shows individual features relative to its absorption, transport, and metabolism.
\end{abstract}

\section{INTRODUCTION}

Of approximately 600 carotenoids that have been characterized from natural sources, 50 show provitamin A activity. In addition to the provitamin $A$ carotenoids, however, many nutritionally inactive carotenoids are absorbed well from the gut and appear in the plasma and tissues (refs. 1-3). Carotenoids are long, highly conjugated, inflexible molecules, most of which are hydrophobic and easily oxidized. Thus, they can either participate in the biological antioxidant network as singlet oxygen quenching and electron-donating molecules or be protected by other antioxidants in biological fluids and tissues. A change in one double bond from a trans to a cis configuration markedly changes the shape of the molecule. In this review, the absorption, transport, and metabolism of carotenoids in humans will be considered with particular attention given to recently published studies. A major conclusion that arises from these studies is that each carotenoid is an individual compound with its own metabolic characteristics within the body.

\section{DIGESTION AND ABSORPTION}

The bioavailability of carotenoids in foods and in commercial preparations varies widely. Only about 5\% of the carotenoids in whole, raw vegetables, for example, is absorbed by the intestine, whereas $50 \%$ or more of the carotenoid is absorbed from micellar solutions. Thus, the physical form in which the carotenoid is presented to intestinal mucosal cells is of crucial importance.

\section{Bile salts}

Bile salts play an essential role both in the absorption of carotenoids and in their subsequent cleavage to vitamin A (refs. 4-6). The concentration of the bile salt, of course, must be above its critical micelle concentration. The solubilization of $\beta$-carotene is proportional to the ratio of dihydroxy- to trihydroxy-bile salts, to the concentration of sodium chloride, and to the $\mathrm{pH}$ but not to the glycine/taurine ratio. The uptake of $\beta$-carotene by intestinal mucosal cells is four times higher when micelles are present than when an emulsion is used. Although the nonionic detergents of the Tween series solubilize $\beta$-carotene well, cellular uptake of $\beta$-carotene is fourfold higher in the presence of bile salts 
(refs. 4-6). Interestingly, retinol uptake by cells is much less affected by the nature of the dispersion (refs, 4-6). The difference in micelle size of the Tween series and of bile salts is particularly interesting (ref. 7). Whereas the micelle size of Tween detergents in isotonic saline is approximately $100,000 \mathrm{~g} /$ aggregate, the conjugated or free bile salts show micelle sizes of 1600 to $11,000 \mathrm{~g} /$ aggregate (ref. 7). Mixtures of nonionic detergents with conjugated or free bile salts give micelles of intermediate size. These physicochemical considerations relate closely to the efficiency of carotenoid absorption by the intestine.

\section{Digestion}

Xanthophyll esters must be hydrolyzed before absorption, and dietary fat greatly stimulates the absorptive process. Whether dietary fat is useful only as a stimulator of the secretion of conjugated bile salts or plays an important role as well in solubilization is not clear. In addition to their solubilizing effects, bile acids may also play a role in the cellular uptake of carotenoids (ref. 6).

\section{B-Carotene}

Because of the possibility that carotenoids play a significant role in reducing the prevalence of some forms of cancer, as well as of other chronic diseases (ref. 8), much attention is currently being given to the absorption, transport, and metabolism of carotenoids in humans. A conventional protocol consists of the following steps: 1) the recruitment of suitable subjects, 2) the provision of lowcarotenoid diets to subjects for a 2- to 8-week period, 3) the administration to subjects of a large (10-50 mg) dose of a carotenoid-rich food or of a selected carotenoid in an appropriate form, usually as a part of a fatty snack, 4) the measurement of plasma carotenoids and retinoids initially and at various times after dosing, 5) correction for endogenous plasma carotenoid concentrations, and 6) calculation of various kinetic parameters.

During the washout period, carotenoid concentrations in plasma fall fairly rapidly for 20 to 30 days and then tend to fall much more slowly (ref. 9). Thus, in the absence of dietary carotenoids, a new steady state is achieved in the plasma that is probably determined, at least in part, by tissue stores of carotenoids. Upon the administration of sizable doses (12-30 $\mathrm{mg}$ ) of $\beta$-carotene, either in a prepared capsule or as vegetables, the $\beta$-carotene concentration in the plasma increases twoto four-fold in most subjects, reaches a maximum at one to two days, and then slowly decreases thereafter (refs. 10, 11). Carotenoids present in commercial beadlets or in oil tend to be utilized more effectively than carotenoids in foods such as carrots, broccoli, and tomato juice.

The absorption of doses of carotenoids is enhanced three- to four-fold when ingested as part of a fatty meal. By a carefully devised balance technique, a dose of $15 \mathrm{mg}$ of $\beta$-carotene in beadlet form was only $15 \%$ absorbed in the absence of a meal but was $47 \%$ absorbed in the presence of a fatty meal (ref. 11).

\section{B-Carotene isomers}

"Betatene", an extract from the alga Dunaliella salina, contains a mixture of $\beta$ carotene, $\alpha$-carotene, and several other carotenoids and lipids. of the $\beta$-carotene, approximately 54\%, 37\%, and $9 \%$ are in the all-trans, 9 -cis, and 13 -cis (plus other isomeric) forms, respectively. When this mixture is given to human subjects, alltrans $\beta$-carotene increases in the plasma of most, but not all, subjects with a maximum between one and two days (ref. 12). It has been noted by many that some individuals respond well to dosing with fairly high carotenoid concentrations in the plasma, whereas others do not. This observation has given rise to the view that humans are divided into two classes, "responders" and "nonresponders." In these studies with "Betatene", 9-cis $\beta$-carotene was not detected in the plasma (ref. 12). Thus, different isomers of the same carotenoid clearly are absorbed and/or are cleared from the plasma at very different rates. 


\section{Other carotenoids}

In the same study with "Betatene" (ref. 12), the change in $\alpha$-carotene concentrations was also studied. Although $\alpha$-carotene is a smaller component of plasma carotenoids than $\beta$-carotene, the absorption and plasma clearance curves were very similar (ref. 12). In this case as well, however, both "responders" and "nonresponders" were noted.

Lycopene in the plasma is present as a mixture of 15 or more isomers (A. Nagao and J.A. Olson, unpublished observations). Two common cis isomers of lycopene, 9-cis and 13 -cis, are enhanced by heat processing (ref. 13). When aciministered to human subjects in tomato juice at a dose of $2.5 \mu \mathrm{mol} / \mathrm{kg} \mathrm{BW}$, the all-trans and the two cis isomers increased to maxima between one and two hours and then declined. On the basis of the relative concentrations of the various isomers in the tomato juice and in the plasma, the authors concluded that the cis isomers were absorbed somewhat better than the all-trans isomer of lycopene (ref. 13). Thus, for $\beta$-carotene, the 9 -cis isomer does not seem to appear in the plasma, whereas, in the case of lycopene, both 9 -cis and 13 -cis seem to be preferentially absorbed.

The di-keto-carotenoid, canthaxanthin, is also absorbed by humans but much less well than $\beta$-carotene (ref. 14). Indeed, $\beta$-carotene and canthaxanthin seem to compete with each other for absorption in humans (ref. 14). Such inhibitory effects of one carotenoid on the absorption of another are probably general.

\section{Model carotenoids}

To reduce the confounding effects of endogenous plasma carotenoids on the in vivo kinetic analysis of carotenoid absorption, three model carotenoids, namely, $\beta-$ apo$8^{\prime}$-carotenal, ethyl $\beta$-apo-8'-carotenoate, and $4,4^{\prime}$-dimethoxy- $\beta$-carotene, were studied in human volunteers. Each subject received a single oral dose of $100 \mu \mathrm{mol}$ of the selected carotenoid in peanut oil as part of a light breakfast. Blood samples were taken before and at various times up to 50 days after dosing (ref. 15).

The three compounds showed quite different kinetic patterns. Ethyl $\beta$-apo-8'carotenoate reached a maximum at 16.5 hours compared with 27.1 hours for $4,4^{\prime}$ dimethoxy- $\beta$-carotene, and from 5 to 11 hours for the metabolites of $\beta$-apo-8'carotenal (ref. 15). The maximum serum concentrations, areas under the curve, and mean sojourn times for the three compounds differed by 50-, 270-, and 5-fold, respectively (ref. 15). In this group of subjects, no "nonresponders" were noted. In summary, the absorption and plasma clearance rates in humans of various carotenoids and their isomers differ markedly.

\section{TRANSPORT}

After their absorption, dietary carotenoids, in large part, are incorporated by intestinal mucosal cells into chylomicra, which are released into the lymph (refs. 16, 17). Chylomicra are digested very rapidly by lipoprotein lipase within the systemic circulation, and the chylomicron remnants are rapidly removed by the liver and other tissues. Very low density lipoproteins (VLDL) next appear as major carriers for carotenoids, and, thereafter, low density lipoproteins (LDL) show the highest concentration of carotenoids in the plasma. Carotenoids also appear in high density lipoproteins (HDL).

Carotenoids are distributed differently among the various lipoprotein classes. Of the total carotenoids present in the plasma of fasting subjects, the VLDL, LDL, and HDL particles contain $14 \%, 55 \%$, and $31 \%$, respectively, of the carotenoids present (ref. 16). Hydrocarbon carotenoids, such as $\alpha$ - and $\beta$-carotene and lycopene, are mainly located in the LDL, whereas the xanthophylls, such as lutein and zeaxanthin, are primarily found in HDL (refs. 16, 17). Thus, the distribution of carotenoids among plasma lipoproteins is roughly similar to that of cholesterol.

The most commonly identified carotenoids in human plasma are lutein, zeaxanthin, $\beta$ cryptoxanthin, lycopene, $\alpha$-carotene, and $\beta$-carotene. With newly developed, high- 
resolution, HPLC systems, 17 or 18 carotenoids plus retinol, retinyl esters, and $\alpha$ and $\boldsymbol{\gamma}$-tocopherol can readily be separated and measured (refs. 18, 19). Indeed, at least an additional 20 carotenoids are present in trace amounts (ref. 19). By the use of HPLC and photodiode array detection, the resolution and identification of carotenoids has been made much easier (ref. 18).

\section{DISTRIBUTION IN TISSUES}

In addition to plasma, carotenoids are found in most other tissues. Although the highest concentrations are present in the adrenal gland, the corpus luteum, and the testes, major storage sites are the liver and adipose tissue (refs. 20-22). In general, the pattern of various carotenoids in tissues reflects that in the serum (refs. 20, 21, 23). This relationship of carotenoid patterns in the serum and tissues does not hold in all cases, however, and, clearly, the ability of some tissues to concentrate carotenoids from the serum indicates that some specialized uptake processes for carotenoids exist.

Carotenoids have been identified in the retina and the pineal gland but not in the brain stem (refs. 21, 24, 25). The macula of the eye has roughly equivalent concentrations of lutein and zeaxanthin but not of other carotenoids (ref. 24). In contrast, the major carotenoid in the pineal gland is $\beta$-carotene ( $r e f .25$ ). The concentrations of various carotenoids in human plasma, however, do not correlate with the presence of macular disease (ref. 26).

Thus, carotenoids tend to be associated with plasma lipoproteins, in a somewhat preferential way, but not apparently with specific carotenoid-binding proteins of plasma. Although carotenoproteins of crustacea have been extensively studied (ref. 27), only one carotenoid-protein complex has been partially characterized from rat liver cytosol (ref. 28). The possible physiological role of this complex has not yet been defined.

\section{METABOLISM}

In nature, the carotenold molecule can be attacked at many points, namely, in the two cyclohexene rings, at various methyl groups, and at various double bonds in the conjugated chain. A wide variety of oxidative and nonoxidative enzymes is involved in catalyzing these reactions.

The manner in which carotenoids are metabolized is very dependent on the species. Thus, fish and birds can convert hydrocarbon carotenoids into oxo-carotenoids, such as astaxanthin and canthaxanthin. Plants employ reversible epoxidation as a protective device for the photosynthetic apparatus (ref. 29). Ways in which mammals and, particularly, humans metabolize various carotenoids, however, are still far from clear. Needless to say, a major focus has been the conversion of $\beta$ carotene and other provitamin A carotenoids into vitamin A.

In 1960, John Glover proposed two possible mechanisms for the formation of retinal from $\beta$-carotene, namely, central fission and asymmetric cleavage (ref. 30). Soon thereafter, an enzyme that cleaved $\beta$-carotene and $\beta$-apo-carotenals to retinal was identified in the cytosol of rat liver and intestine (refs. 31, 32). The partially purified enzyme is cytosolic, requires molecular oxygen, possesses essential sulfhydryl groups, is inhibited by metal-binding reagents, and requires a detergent. The enzyme produces retinal as the major, if not sole, product from $\beta$ carotene and other substrates (refs. 33-35). The cleavage enzyme, which is termed $\beta$-carotenoid-15,15'-dioxygenase, is also found in tissues of the newborn (ref. 36).

In addition to the central cleavage pathway, asymmetric cleavage to yield $\beta$-apocarotenals has also been shown in human intestinal tissue (refs. 37-39). The enzyme or enzymes responsible for asymmetric cleavage, however, have not yet been purified or characterized. Although the action of dioxygenases on hydrocarbon carotenoids will exclusively yield aldehydic cleavage products, retinoic acid can also be produced from $\beta$-carotene, presumably without retinal as a free intermediate (refs. 38,40 ). The mechanism by which this reaction occurs has not yet been clarified. 
The metabolism of other provitamin A carotenoids has been much less studied; presumably, they are converted into vitamin A, either by central or asymmetric cleavage. Even less is known about the metabolism of nutritionally inactive carotenolds, such as lycopene, lutein, and zeaxanthin.

of the model carotenoids, ethyl $\beta$-apo-8'-carotenoate is not detectably metabolized in humans (ref. 15). On the other hand, $4,4^{\prime}$-dimethoxy- $\beta$-carotene is demethylated slowly and then is oxidized through several possible intermediates to canthaxanthin (ref. 15). $\beta$-Apo-8'-carotenal is very actively metabolized in the intestine (being both oxidized to the corresponding acid and reduced to $\beta$-apo-8'-carotenol, which is then esterifled with long chain fatty acids (ref. 15)). Thus, as with their absorption, carotenoids tend to follow individual pathways of metabolism that depend on their specific structures.

\section{EXCRETION}

Dietary carotenoids that are not absorbed, of course, are excreted in the feces. Some biliary metabolites of carotenoids may also appear in the feces, together with any carotenoids present in sloughed mucosal cells. Although polar metabolites of carotenoids, probably in conjugated form, are most likely excreted in the urine, little is known of the nature of these compounds. Endogenous carotenoids in skin are significantly increased when large amounts of $\beta$-carotene are ingested (ref. 41). Thus, some carotenoids will be lost by the sloughing of keratinized skin cells. The oxidation of carotenoids in whole or in part to carbon dioxide is a relatively minor reaction $(\langle 5 \%)$, at least in rats ( $r e f .30$ ), and probably also in humans.

\section{CONCLUDING REMARKS}

Humans metabolize carotenoids in quite different ways from most other species. Although some newly developed animal models, such as the ferret and the preruminant calf, absorb intact carotenoids much better than rodents and other like experimental animals do, studies in humans are still preferable. Thus, the development of new procedures, such as the use of carotenoids labelled with heavy isotopes for studying the absorption and metabolism of carotenoids in humans, is well worth pursuing. With current interest in the non-nutritional activities of carotenoids, in relation to their possible protective effects against chronic disease, the need exists to understand better their absorption, transport, tissue distribution, and metabolism. What seems to have emerged from recent studies of carotenoids in humans is that each carotenoid is handled in quite an individual fashion, not only in regard to its rates of absorption and plasma clearance, but also in the nature of the metabolites formed. Clearly, we have only begun to understand the dynamics of carotenoids in humans and their potential protective actions.

\section{ACKNOWLEDGMENTS}

This work was supported in part by grants DK-39733, CA-46406, and HD-27994 from the National Institutes of Health, Bethesda, MD; by grant 02808-2 from the Thrasher Research Fund, Salt Lake City, UT; and by support from the Iowa State Agriculture and Home Economics Experiment Station, paper no. J-15412, project no. 3035.

\section{REFERENCES}

1. J.A. Olson, in Handbook of Vitamins (L.J. Machlin, ed.), pp. 1-57, Marcel Dekker, New York (1990).

2. J.A. Olson, in Modern Nutrition in Health and Disease (M.E. Shils, J.A. Olson and M. Shike, eds.) 8 edn., pp. 287-307, Lea \& Febiger, Philadelphia (1993).

3. 0 . Isler (ed.) Carotenoids, Birkhäuser Verlag, Basel (1971).

4. J.A. Olson, J, Lipid Res., 5, 402-408 (1964).

5. M. El-Gorab and B.A. Underwood, Biochim. Biophys. Acta, 306, 58-66 (1973).

6. M.J. El-Gorab, B.A. Underwood and J.D. Loerch, Biochim. Biophys. Acta, 401, 
$265-277$ (1975).

7. J.A. Olson and J.S. Herron, Abstr. Sixth Intern, Cong. Biochem., New York, p. 588 (1964).

8. V.N. Singh, in Lipid-Soluble Antioxidants: Biochemistry and Clinical Applications (A.S.H. Ong and L. Packer, eds.), pp. 208-227, Birkhäuser Verlag, Basel (1992).

9. C.L. Rock, M.E. Swendseid, R.A. Jacob and R.W. McKee, J. Nutr., 122, 96-100 (1992).

10. E.D. Brown, M.S. Micozzi, N.E. Craft, J.G. Bieri, G. Beecher, B.K. Edwards, A. Rose, P.R. Taylor and J.C. Smith Jr. Am, J. Clin. Nutr., 49, 1258-1265 (1989).

11. P.E. Bowen, S. Mobarhan and J.C. Smith Jr., Methods Enzymol., 214, 3-17 (1993).

12. W. Stahl, W. Schwarz and H. Sies, J. Nutr., 123, 847-851 (1993).

13. W. Stahl and H. Sies, J. Nutr., 122, 2161-2166 (1992).

14. P.E. Bowen, M. Stacewicz-Sapuntzakis, M. Sawicki, J.W. Erdman and W.S. White, FASEB J., 6, A1646 (1992).

15. S. Zeng, H.C. Furr and J.A. Olson, Am. J. Clin. Nutr., 56, 433-439 (1992).

16. B.A. Clevidence and J.G. Bieri, Methods Enzymol., 214, 33-46 (1993).

17. D.G. Cornwe11, F.A. Kruger and H.B. Robinson, J. Lipid Res., 3 , 65-70 (1962) .

18. A.B. Barua, D. Kostic and J.A. Olson, J. Chromatogr., in press.

19. F. Khachik, G.R. Beecher, M.B. Goli, W.R. Lusby and J.C. Smith Jr., Anal, Chem. , 64, 2111-2122 (1992).

20. L.A. Kaplan, J.M. Lau and E.A. Stein, Clin. Physiol. Biochem., $\underline{8}, 1-10$ (1990).

21. W. Stahl, W. Schwartz, A.R. Sundquist and H. Sies, Arch. Biochem. Biophys., 294, 173-177 (1992).

22. H.H. Schmitz, C.L. Poor, R.B. Wellman and J.W. Erdman Jr., J.Nutr., 121, 1613-1621 (1992).

23. R.S. Parker, J. Nutr., 119, $101-104$ (1989).

24. G.J. Handelman, E.A. Dratz, C.C. Reay and F.J.G.M. Van Kuijk, Invest. Ophthalmol. Visual Sci., 29, 850-855 (1988).

25. H. Shi, H.C. Furr and J.A. Olson, Brain Res. Bul1., 26, 235-239 (1991)

26. T.A.B. Sanders, A.P. Haines, R. Wormald, L.A. Wright and O. Okeid, Am. J. Clin, Nutr., 57, $428-433$ (1993).

27. P.F. Zagalsky, E.E. Eliopoulos and J.B.C. Findlay, Comp. Biochem. Physiol., 97B, $1-18$ (1990)

28. M.R. Lakshman and C. Okoh, Methods Enzymol. , 214, 74-86 (1993).

29. A. Bendich and J.A. Olson, FASEB J., 3, 1927-1932 (1989).

30. J. Glover, Vitam. Horm., 18, 371-386 (1960).

31. D.S. Goodman and H.S. Huang, Science, $149,879-880$ (1965).

32. J.A. Olson and O. Hayaishi, Proc. Nat. Acad. Sci. USA, 54, 1364-1370 (1965).

33. J.A. Olson, J. Nutr., 119, 105-108 (1989).

34. M.R. Lakshman, I. Mychkovsky and M. Attlesey, Proc. Nat. Acad. Sci. U.S.A., 86, $9124-9128$ (1989).

35. M.R. Lakshman and C. Okoh, Methods Enzymol., 214, 256-269 (1993).

36. M.R. Lakshman, L.H. Johnson, C. Okoh, M. Attlesey, I. Mychkovsky and H.N. Bhagavan, J. Nutr. Biochem., in press.

37. X.D. Wang, G.W. Tang, J.G. Fox, N.I. Krinsky and R.M. Russell, Arch. Biochem. Biophys. , 285, 8-16 (1991).

38. G.W. Tang, X.D. Wang, R.M. Russe11 and N.I. Krinsky, Biochemistry, 30, 98299834 (1991).

39. X.D. Wang, N.I. Krinsky, G.W. Tang and R.M. Russell, Arch. Biochem. Biophys., 293, 298-304 (1992).

40. J.L. Napoli, Methods Enzymol., 214, 193-202 (1993).

41. M.R. Prince and J.K. Frisoli, Am. J.Clin. Nutr., 57, 175-181 (1993). 